\title{
The Forming Theory and Computer Simulation of the Rotary Cutting Tools with Helical Teeth and Complex Surfaces
}

\author{
Huran Liu \\ Department of Mechanical Engineering \\ Zhejiang University of Science and Technology \\ Hangzhou 310023, China
}

\section{Abstract}

This paper researched the forming theory of the cutting tools with helical teeth and complex surfaces. Deduced the nonlinear equations of the movement of NC system to generate such tools, presented the way to find the solution of the equations, calculated the cross section graphics of the teeth profile and computer simulation of NC machining.

Keywords: rotary cutting tools, Helical teeth, Computer simulation

\section{The cutting edges of the rotary tools with helical teeth}

Fig. 1 showed a rotary tool with complex surfaces. The coordinate of any point on the cutting edge:

$$
x_{P A}^{(1)}=x_{P A}, y_{P A}^{(1)}=r_{P A} \cos \left(\psi_{A}+\varphi\right), z_{P A}^{(1)}=r_{P A} \sin \left(\psi_{A}+\varphi\right)
$$

Where $r_{p}$ : the rotary radius of any point $\mathrm{P}$

$$
\psi_{A} \text { : The position angle of the radius line relate to the xoy plan. }
$$

From derivative geometry, we know that $\psi$ should be satisfied by the following equation

$$
\frac{d \psi_{A}}{d x_{P A}}=\frac{\operatorname{tg} \beta}{r_{P A}} \sqrt{1+\left(\frac{d r_{P A}}{d x_{P A}}\right)^{2}}
$$

Where $\beta$ : the helical angle of cutting edge. The tangent vector of cutting edge:

$$
t_{P A x}^{(1)}=\frac{d x_{P A}^{(1)}}{d x_{P A}}=1
$$

$t_{P A z}^{(1)}=\frac{d z_{P A}^{(1)}}{d x_{P A}}=\frac{d r_{P A}}{d x_{P A}} \sin \left(\psi_{A}+\varphi\right)+r_{P A} \frac{d \psi_{A}}{d x_{P A}} \cos \left(\psi_{A}+\varphi\right)$

If $x_{p_{A}}, r_{p A}, \psi_{A}$ are substituted by $x_{p_{B}}, r_{p B}, \psi_{B}+2 \pi / z$, equation (1) will be the coordinates of the two cutting edged $\mathrm{A}$ and $\mathrm{B}$, which are in the neighborhood with each other, respectively. And so are the Eqs (3), after rotate an angle $\phi$ about axis $x, \psi_{A} \quad$ should be substituted by $\quad \psi_{B}+2 \pi / z . \quad z$ represents the number of teeth.

\section{2. the establishment of the coordinate system}

In the Fig.2, a grinder is generating a rotary cutting tool. Two coordinate system need to be established.

1) The fixed coordinate system $O x y z$

The $x$ - axis of this system is the rotation axis of the work; the original point $\mathrm{O}$ of this system is at the rotation center of the larger end of the work.

2) Grinder coordinate system $O x y z$

The original point $O s$ is at the center of grinder wheel, the $y_{s}$ axis is parallel to $y$-axis, the angle between $x_{s}$ and $x$ equal 
to $\pi / 2-\Sigma$. The coordinates of point $O s$ in fixed system are $x_{c}, y_{c}, z_{c}$. The coordinate transformation of two systems can be state as

$$
\left[\begin{array}{l}
x \\
y \\
z
\end{array}\right]=\left[\begin{array}{ccc}
\sin \Sigma & 0 & \cos \Sigma \\
0 & 1 & 0 \\
-\cos \Sigma & 0 & \sin \Sigma
\end{array}\right]\left[\begin{array}{l}
x_{s} \\
y_{s} \\
z_{s}
\end{array}\right]+\left[\begin{array}{l}
x_{c} \\
y_{c} \\
z_{c}
\end{array}\right]
$$

\section{The surface equation and normal vector of grinder}

The surface of grinder is a circular cone, let $\mathrm{M}_{\mathrm{b}}$ represent any point on the cone, the coordinates in local system of $\mathrm{M}_{\mathrm{b}}$ can be expressed as following ( see Fig.2)

$$
x_{M B}^{(S)}=-\left(R-r_{m B}\right) \operatorname{tg} \alpha_{B}, y_{M B}^{(S)}=-r_{m B} \cos \theta_{B}, z_{M B}^{(S)}=r_{M B} \sin \theta_{B}
$$

Where $r_{m b}$ : The radius of point $M_{b}$

$\theta_{b}$ : The angle position parameter

$\alpha_{b}$ : The bottom angle of the grinder

The coordinates in the fixed system are

$x_{M B}^{(0)}=-\left(R-r_{M B}\right) \operatorname{tg} \alpha_{B} \sin \varepsilon+r_{M B} \sin \theta_{B} \cos \varepsilon+x_{c}$

$$
y_{M B}^{(0)}=-r_{M B} \cos \theta_{B}+y_{c}
$$

$z_{M B}^{(0)}=\left(R-r_{M B}\right) \operatorname{tg} \alpha_{B} \cos \varepsilon+r_{M B} \sin \theta_{B} \sin \varepsilon+z_{c}$

The normal vector in the local system is:

$$
n_{M B}^{(S)}\left(-\cos \alpha_{B},-\sin \alpha_{B} \cos \theta_{B}, \sin \alpha_{B} \sin \theta_{B}\right)
$$

The normal vector in the fixed system is:

$$
n_{M B}^{(0)}=\left(-\cos \alpha_{B} \sin \Sigma+\sin \alpha_{B} \sin \theta_{B} \cos \Sigma,-\sin \alpha_{B} \cos \theta_{B}, \cos \alpha_{B} \cos \Sigma+\sin \alpha_{B} \sin \Sigma\right)
$$

Let $M a$ represent any point at the largest circle of the grinder, the coordinate of point $M a$ in local system can be expressed as following:

$$
x_{M A}^{(S)}=0, y_{M A}^{(S)}=-R \cos \theta_{A}, z_{M A}^{(S)}=R \sin \theta_{A}
$$

Where R: the radius of the largest circle of the grinder

$$
\theta_{A}: \text { The angle position parameter }
$$

The coordinates of the same point in the fixed system are

$$
x_{M A}^{(0)}=R \sin \theta_{A} \cos \varepsilon+x_{c}, y_{M A}^{(0)}=-R \cos \theta_{A}+y_{c}, z_{M A}^{(0)}=R \sin \theta_{A} \sin \varepsilon+z_{c}
$$

\section{The moving equations of NC system}

The problem to be solved by this thesis is: when use a grinder with curtain shap to generate the teeth of the cutter, the two neighborhood cutting edges A and B should be made out by the two sides of the grinder in the same time, so that, the relative movement of the work and grinder should satisfy special mathematical and geometrical relationship.

Suppose that, the contact points on B-side of grinder and cutting edge B of work are $\mathrm{M}_{b}$ and $\mathrm{P}_{b}$ respectively. The coordinates in the fixed system should be the same

$$
x_{m b}=x_{p b}, y_{m b}=y_{p b}, z_{m b}=z_{p b}
$$

Following relation can be derived from Esq. (1) and Esq. (6)

$$
\begin{aligned}
x_{p b}+\left(R-r_{M B}\right) \operatorname{tg} \alpha_{B} \sin \Sigma-r_{M B} \sin \theta_{B} \cos \Sigma & =x_{c} \\
y_{m b} & +r_{M B} \cos \theta_{B}=y_{c}
\end{aligned}
$$

$z_{m b}-\left(R-r_{M B}\right) \operatorname{tg} \alpha_{B} \cos \Sigma-r_{M B} \sin \theta_{B} \sin \Sigma=z_{c}$ 
The normal vector of the grinder at $\mathrm{Mb}$ should be vertical to the tangent vector of the cutting edge at $\mathrm{Pb}$, so that

$$
n_{m b} \bullet t_{p b}=0
$$

From Esq.(3) and Esq.(8), we obtain:

$$
\left(t_{p b z} \sin \Sigma+\cos \Sigma\right) \sin \theta_{b} \operatorname{tg} \alpha-t_{p b y} \cos \theta_{b} \operatorname{tg} \alpha-\left(\sin \Sigma-t_{p b z} \cos \Sigma\right)
$$

Suppose that the contacting points of the largest circle A and cutting edge $a$ are $M a$ and $P a$ respectively, the coordinates of such two points in the fixed system should be the same:

$$
x_{m a}=x_{p a}, y_{m a}=y_{p a}, z_{m a}=z_{p a}
$$

From Esq.(1) and Esq.(9), we obtain:

$$
x_{c}=x_{p a}-R \sin \theta_{a} \cos \Sigma, y_{c}=r_{p a} \cos \left(\psi_{a}+\phi\right)-R \cos \theta_{a}, \quad z_{c}=r_{p a} \sin \left(\psi_{a}+\phi\right)-R \sin \theta_{a} \sin \Sigma
$$

At the same time, on the same grinder, the position of the center point C should be single. From Esq.(12) and Esq.(16), following equation can be derived.

$$
\begin{gathered}
x_{M B}+\left(R-r_{M B}\right) \operatorname{tg} \alpha_{B} \sin \varepsilon-r_{M B} \sin \theta_{B} \cos \varepsilon=x_{p a}-R \sin \theta_{a} \cos \Sigma \\
y_{M B}^{(0)}+r_{M B} \cos \theta_{B}=r_{p a} \cos \left(\psi_{a}+\phi\right)-R \cos \theta_{a}
\end{gathered}
$$

While, during the generation, the coordinate Zc remain unchanged, so that:

$$
\begin{array}{r}
z_{c}=r_{p a} \sin \left(\psi_{a}+\phi\right)-R \sin \theta_{a} \sin \Sigma \\
z_{m b}-\left(R-r_{M B}\right) \operatorname{tg} \alpha_{B} \cos \Sigma-r_{M B} \sin \theta_{B} \sin \Sigma=z_{c}
\end{array}
$$

Esq. (14)(17)(18)(19), and (20) form a nonlinear equation system with five equations, In these equations there are 6 unknown parameters: $\theta_{a}, \theta_{b}, x_{p a}, x_{p b}, r_{m b}, \phi$. One of the parameters can be given before hand, so the equations are solvable.

\section{Example}

There is a rotary tool with the outside surface of a transected cone, as showed in Fig.4. The main parameters of the tool areas following:

The maximum diameter: $\mathrm{d}=10 \mathrm{~mm}$

The length of the cutter: $\quad 1=24.5 \mathrm{~mm}$

The number of teeth: $\quad z=20$

The tape angle: $\quad \xi=7^{\circ}$

The helical angle: $\beta=20^{\circ}$

The parameters of the grinder are as following:

The diameter of the grinder: $\mathrm{D}=80 \mathrm{~mm}$

The bottom angle of the grinder: $\alpha_{b}=60^{\circ}$

The parameters of installation are listed below:

$\mathrm{Zc}=0$

Installation angle: $\Sigma=20^{\circ}$

Through computer calculation, the coordinates of the grinder center during generation can be solved out and listed bellow. (Deleted)

\section{The computer modeling and simulation of the generation of the rotary tools}

In generation, the relative displacement of the grinder and the work is very complicate. Only by numerical control operator can realize such motion requirement. For testifying the theoretical deduction and numerical calculation, we used the imported I-DEAS software to simulate the cutting movement of the work and the grinder at the SUN-CAD workstation. This is the first time to use computer solid modeling in research of the rotary tools with complex surfaces, and we made the best use of the new achievement in computer graphics, and computer Aid Design at home and abroad.

The I-deas soft ware system contains 5 families:1)solid modeling, 2)system assembly,3)engineering analysis,4)system 
dynamics and 5)drafting. The research of this thesis used two of them: solid modeling (Geomod) and engineering drafting( Geodraw)

First, create the object of the work and grinder, according to their shapes, and stored them with Geomod; secondly, made grinder and work to move relate to each other; than do the Boolean operation, at every position. The generation of the work corresponds to "cut object" operator, one of the Boolean calculation. Finally, after the grinder goes through all move positions the teeth profile of the work was formed.

In the beginning, we choose hand operation, by select menu with mouse, let the grinder and work go several steps according to the required replacement, and recorded our operation into a program file. A passage of the I-deas program corresponds to one cutting step is as follows:

Return to main menu---get the stored work----orient it this time----rotate about its axes---give the value of rotate angle---store it the second time---get the stored grinder---translate it along $\mathrm{x}$ and y direction respectively ---give the value of the translate amount ---store the grinder once more ---return to main menu again--- select Boolean operation ---select cutting operation ---define the cutter, the grinder---define the object to be cut, the work---store the new cutter work the third time

In running this short passage of program, we can see from the sceen of the computer a series of pictures in consequence:

The original shape of the work;

The original position of the grinder;

The rotation of the work;

The translation of the grinder relate to the work;

The grinder in cutting with the work;

The shape and the size of the chip formed from cutting, it is growing larger and larger every moment;

The work cut is waiting for further cutting;

Than, let the Fortran program, with which the coordinates of grinder center were calculated, to output automatically a passage of program as mentioned above, whenever a displacement is calculated. Every movement is corresponding to one passage of I-deas format exactly. In this way, the whole generate progress can be simulated. The shape of the work with one groove showed in Fig.3. The sectional curvature of the tooth profile showed in Fig.4.

\section{Conclusions}

From the analysis and research mentioned above, following conclusions can be arrived at:

1) The forming theory of the rotary tools with helical teeth and complex surfaces is correct. To generate such a kind of tools, three coordinates numerical control manufacturing system is needed.

3) From computer simulation, we can testify the formulas of theoretic deduction and result of numerical calculation presented in this paper.

We can examine if the interference and other problem. Which are often caused by incorrect parameter selection, would take place and the way to prevent it before hand.

\section{References}

Bao Qingshan, A study on a virtual manufacturing model of a revolving milling cutter in 2-axis numerical control processing. J Mater Process Tech, 2000,120(1-3):68-75.

Chen chaoguang, A manufacturing model of carbide-tipped spherical milling cutter. Proc.Instu. Mech. Engrs., 1999,213(B)713-724. 


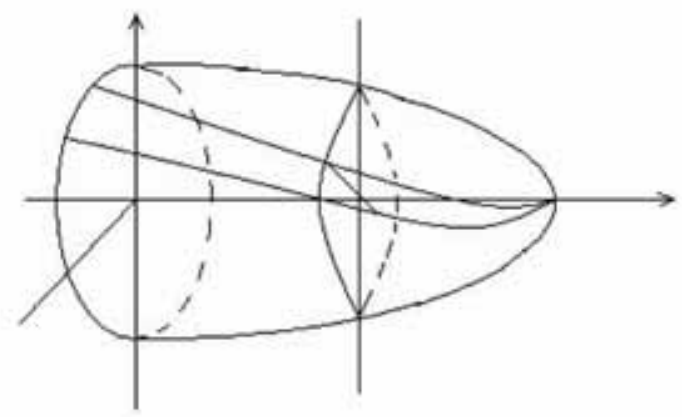

Figure 1

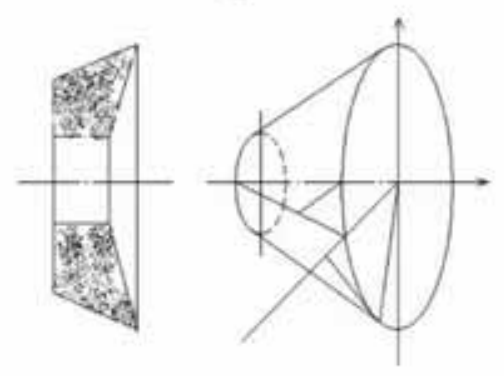

Figure 2

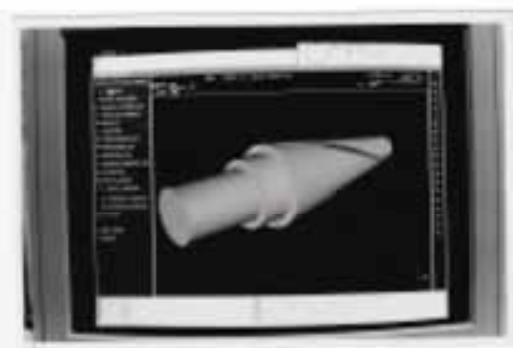

Figure 3. computer simulation of the rotary cutting tools

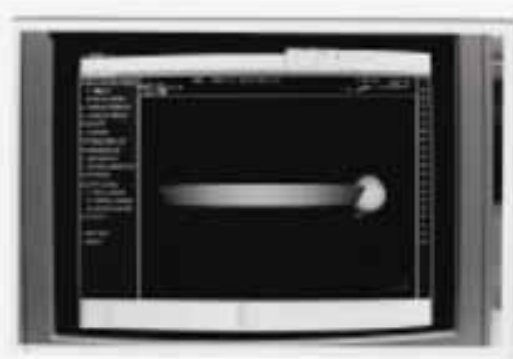

Figure 4. computer simulation of the rotary cutting tools 